\title{
Erratum to: Frosted branch angiitis complicated by retinal vein occlusion: clinical course and long-term visual outcome
}

\author{
Gabriel Greifner $^{1} \cdot$ Piergiorgio Neri ${ }^{2} \cdot$ Radgonde Amer ${ }^{1}$
}

Published online: 23 January 2016

(C) Springer-Verlag Berlin Heidelberg 2016

Erratum to: Graefes Arch Clin Exp Ophthalmol

DOI 10.1007/s00417-015-3255-0

The original version of this article inadvertently contained mistake.

Reason: incorrect spelling of author's name

Corrected version: Gabriel Greifner, Piergiorgio Neri, Radgonde Amer

The online version of the original article can be found at http://dx.doi.org/ 10.1007/s00417-015-3255-0.

\footnotetext{
Radgonde Amer

radgonde@gmail.com

1 Department of Ophthalmology, Hadassah-Hebrew University Medical Center, POB 12000, Jerusalem 91120, Israel

2 Department of Ophthalmology, Polytechnic University of Marche, Ancona, Italy
} 